\title{
Interpreting Dose-Response Relation for Exposure to Multiple Sound Impulses in the Framework of Immunity
}

\author{
Hongyun Wang1, Wesley A. Burgei ${ }^{2}$, Hong Zhou ${ }^{3 *}$ \\ ${ }^{1}$ Department of Applied Mathematics and Statistics, University of California, Santa Cruz, CA, USA \\ ${ }^{2}$ US Department of Defense, Joint Non-Lethal Weapons Directorate, Quantico, VA, USA \\ ${ }^{3}$ Department of Applied Mathematics, Naval Postgraduate School, Monterey, CA, USA \\ Email: ‘hzhou@nps.edu
}

How to cite this paper: Wang, H., Burgei, W.A. and Zhou, H. (2017) Interpreting Dose-Response Relation for Exposure to Multiple Sound Impulses in the Framework of Immunity. Health, 9, 1817-1842. https://doi.org/10.4236/health.2017.913132

Received: October 8, 2017

Accepted: December 17, 2017

Published: December 20, 2017

Copyright @ 2017 by authors and Scientific Research Publishing Inc. This work is licensed under the Creative Commons Attribution International License (CC BY 4.0).

http://creativecommons.org/licenses/by/4.0/

C) (i) Open Access

\begin{abstract}
Hearing loss is a common military health problem and it is closely related to exposures to impulse noises from blast explosions and weapon firings. In a study based on test data of chinchillas and scaled to humans (Military Medicine, 181: 59-69), an empirical injury model was constructed for exposure to multiple sound impulses of equal intensity. Building upon the empirical injury model, we conduct a mathematical study of the hearing loss injury caused by multiple impulses of non-uniform intensities. We adopt the theoretical framework of viewing individual sound exposures as separate injury causing events, and in that framework, we examine synergy for causing injury (fatigue) or negative synergy (immunity) or independence among a sequence of doses. Starting with the empirical logistic dose-response relation and the empirical dose combination rule, we show that for causing injury, a sequence of sound exposure events are not independent of each other. The phenomenological effect of a preceding event on the subsequent event is always immunity. We extend the empirical dose combination rule, which is applicable only in the case of homogeneous impulses of equal intensity, to accommodate the general case of multiple heterogeneous sound exposures with non-uniform intensities. In addition to studying and extending the empirical dose combination rule, we also explore the dose combination rule for the hypothetical case of independent events, and compare it with the empirical one. We measure the effect of immunity quantitatively using the immunity factor defined as the percentage of decrease in injury probability attributed to the sound exposure in the preceding event. Our main findings on the immunity factor are: 1) the immunity factor is primarily a function of the difference in SELA (Aweighted sound exposure level) between the two sound exposure events; it is
\end{abstract}


virtually independent of the magnitude of the two SELA values as long as the difference is fixed; 2) the immunity factor increases monotonically from 0 to $100 \%$ as the first dose is varied from being significantly below the second dose, to being moderately above the second dose. The extended dose-response formulation developed in this study provides a theoretical framework for assessing the injury risk in realistic situations.

\section{Keywords}

Risk of Significant Injury, Logistic Dose-Response Relation, SELA (A-Weighted Sound Exposure Level), Effective SELA for Multiple Heterogeneous Sound Impulses, Fatigue and Immunity

\section{Introduction}

Hearing loss is the third most common health problem in the US and more than 28 million Americans have lost some hearing. There are three basic types of hearing loss: conductive hearing loss, sensorineural hearing loss and mixed hearing loss [1]. This classification is based on which part of the auditory system is damaged. Conductive hearing loss occurs when there is damage to the eardrum and the tiny bones of the middle ear. It may be caused by ear infection or impacted earwax and it results in a reduction in the ability to hear faint sounds. Luckily, conductive hearing loss can often be medically or surgically reversed. Sensorineural hearing loss stems from damage to the inner ear or auditory nerve. For people with sensorineural hearing loss, sound appears unclear or muffled. Unfortunately, sensorineural hearing loss is permanent. It cannot be corrected but patients with sensorineural hearing loss can be helped through the use of hearing aids. When a conductive hearing loss occurs together with a sensorineural hearing loss, the hearing loss is called a mixed hearing loss. Symptoms of mixed hearing loss include sounds turning softer in volume and becoming more difficult to understand. One possible cause of sensorineural hearing loss is exposure to loud noise or blast, which is very common in a battlefield. In fact, hearing loss is one of the most prevalent military medical problems. According to the US Department of Veterans Affairs [2] [3], in 2014 more than 933,000 Veterans have hearing loss disability, and nearly 1.3 million experiencing tinnitus which refers to the perception of a ringing, buzzing, or other kind of noise in the ears [4]. With an increasing trend in hearing loss among veterans, it is important to be able to assess the risk of significant hearing loss injury when exposed to blast explosions [5].

The impacts of sound waves on humans are complicated and they depend on the frequency, sound pressure level, and duration. In [6] Dr. Chan and co-workers assembled experimental data of chinchillas exposed to multiple impulse noise shots in the laboratory [7]. They chose chinchillas to model human hearing response because the size, structure, and function of the chinchilla's ear are very 
similar to those of humans. They used the A-weighted sound exposure level (SELA) as an effective single metric (the dose) for predicting the injury risk [8]. Based on the test data they constructed a logistic dose-response relation for unprotected human ears. They adopted an empirical dose combination rule to combine multiple identical sound impulses, uniformly distributed in time, into one effective combined dose [6] [9]. The injury risk over the combined event (i.e., over the sequence of multiple noise shots) is governed by the dose-response relation with the effective combined SELA as the dose.

They also validated the dose-response curve against historical human data from rifle noise tests [10] and proposed a temporary threshold shift (TTS) recovery model in the form of a log-linear function.

In this paper, we carry out a mathematical analysis based on the dose-response relation and the dose combination rule developed in [6]. The motivations of our analysis are: 1) to understand the risk of hearing loss injury caused by multiple noise impulses from the point of view of individual sound exposure events; 2) to extend the dose combination rule developed in [6] to accommodate the general case where we need to combine multiple heterogeneous impulses of non-uniform SELA values into one effective combined SELA value. In the framework of the dose-response relation and the dose combination rule, we examine the synergy for causing injury (fatigue) or negative synergy (immunity) or independence among sound exposure events. The sign and magnitude of synergy will shed light on the role of acoustic reflex (which tends to decrease the injury risk for subsequent sound exposures) and on the role of fatigue/partial damage (which tends to increase the injury risk for subsequent sound exposures). Our primary goal is to establish a unified dose-response formulation for assessing the injury risk of unprotected ears caused by a heterogeneous sequence of sound exposures with non-uniform SELA values. This extended dose-response formulation will provide the foundation for assessing the injury risk in realistic situations.

We organize the rest of the paper as follows. In Section 2 we review the relevant results in [6]. Section 3 contains an analysis of unconditional injury probabilities and conditional probabilities as governed by the dose-response relation and by the dose combination rule, with the goal of determining whether the interactive effect among sequential doses is immunity or fatigue. In Section 4, we extend the dose combination rule to the general case of a heterogeneous sequence of sound exposure events with non-uniform doses. We then construct the dose combination rules for the case of independent events and we compare the two rules. In Section 5, we investigate quantitatively the effect of immunity measured by the immunity factor defined as the percentage of decrease in the injury probability attributed to the sound exposure in the preceding event. We carefully work through the detailed behaviors of the immunity factor in four regimes of parameter values. Finally, we summarize the extended dose-response formulation and the main conclusions in Section 6, and provide a mathematical proof of a theorem in the Appendix. 


\section{Review of Relevant Results in [6]}

In [6], experimental data of permanent threshold shift (PTS) after exposure to multiple impulse noise shots was studied. A dose-response relation was constructed for PTS of various cut-off levels. The empirical dose-response relation is expressed in the form of a logistic model:

$$
P\left(\text { SELA }_{\text {comb }}\right)=\frac{1}{1+\exp \left(-\alpha\left(\text { SELA }_{\text {comb }}-\text { ID }_{50}\right)\right)}
$$

In the dose-response relation (1):

- SELA $_{\text {comb }}$ is the combined A-weighted sound exposure level, a combined single metric quantifying the overall effect of multiple impulse shots;

- $P$ is the probability of PTS of a given cut-off level (for example, PTS $>40$ $\mathrm{dB})$;

- $\quad \mathrm{ID}_{50}$ denotes median injury dose at which the injury risk $P$ is $50 \%$; and

- $\alpha$ is the coefficient describing the steepness of the curve around the median injury dose.

In [6], parameters $\operatorname{ID}_{50}$ and $\alpha$ were determined from experimental data for individual PTS cut-off levels ranging from $1 \mathrm{~dB}$ to $70 \mathrm{~dB}$. It was observed that the steepness coefficient $\alpha$ remains approximately constant around $\alpha=0.1 /(\mathrm{dBA})$ for the whole range of PTS cut-off levels. The median injury dose $\mathrm{ID}_{50}$, however, increases monotonically with respect to the PTS cut-off level. It goes from $\mathrm{ID}_{50}=136 \mathrm{dBA}$ at $1 \mathrm{~dB}$ PTS cut-off to $\mathrm{ID}_{50}=212 \mathrm{dBA}$ at $70 \mathrm{~dB}$ PTS cut-off. For $40 \mathrm{~dB}$ PTS cut-off, $\mathrm{ID}_{50}=170 \mathrm{dBA}$.

Figure 1 shows the dose-response relations for PTS cut-off levels ranging from $5 \mathrm{~dB}$ to $60 \mathrm{~dB}$, based on parameter values from [6]. The thick gray curve

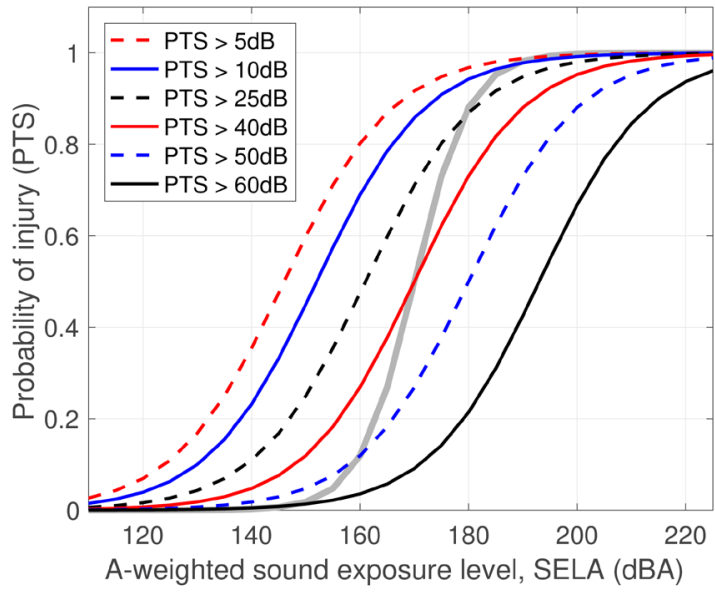

Figure 1. Permanent threshold shift (PTS) dose-response relations. The dose is the A-weighted sound exposure level (SELA); the response is the magnitude of PTS (a random variable), represented as the probability of PTS above a given cut-off level. The dose-response relations are plotted, respectively, for PTS cut-off levels $5 \mathrm{~dB}, 10 \mathrm{~dB}, 25 \mathrm{~dB}$, $40 \mathrm{~dB}, 50 \mathrm{~dB}$, and $60 \mathrm{~dB}$, using parameter values from [6]. The thick gray curve is for $\alpha=0.2 /(\mathrm{dBA})$ and $\mathrm{ID}_{50}=170 \mathrm{dBA}$, demonstrating the effect of doubling steepness coefficient $\alpha$. 
depicts a hypothetical dose-response relation with $\alpha=0.2 /(\mathrm{dBA})$, twice the value from experimental data [6]. It illustrates the effect of steepness coefficient $\alpha$.

In this study, we define injury as PTS above $40 \mathrm{~dB}$, which has a median injury dose of $170 \mathrm{dBA}$. In the subsequent analysis, we shall use $\mathrm{ID}_{50}=170 \mathrm{dBA}$ unless specified otherwise.

In the dose-response relation (1), the dose is quantified by SELA comb $_{\text {. For a }}$ single shot (one impulse), the sound exposure (SE) is defined as the time integral of squared A-weighted sound pressure:

$$
E=\int(\text { A-weighted } p(t))^{2} \mathrm{~d} t
$$

Conventionally, the sound exposure in the air is measured relative to the reference sound exposure $E_{0} \equiv 400(\mu \mathrm{Pa})^{2} \cdot \mathrm{s}$. The sound exposure level (SELA) expresses the sound exposure in the unit of $\mathrm{dBA}$ as

$$
\begin{aligned}
\text { SELA } & =10 \log _{10}\left(\frac{E}{E_{0}}\right) \mathrm{dBA} \\
& =10 \log _{10}\left(\frac{\int(\text { A-weighted } p(t))^{2} \mathrm{~d} t}{400(\mu \mathrm{Pa})^{2} \cdot \mathrm{s}}\right) \mathrm{dBA}
\end{aligned}
$$

For multiple shots of equal intensity, the effective combined SELA for $N$ shots is calculated using the dose combination rule described in [6]:

$$
\mathrm{SELA}_{\text {comb }}=\mathrm{SELA}+\lambda \log _{10} N
$$

where $N$ is the number of impulses, and SELA is sound exposure level of each individual impulse. Here "effective combined SELA" or "dose combination" means "combining multiple sound exposure events into one composite event with an effective combined SELA value as a single metric quantifying the overall effect of multiple sound exposure events". We call this "dose combination" instead of "dose accumulation" to distinguish it from the situation where the dose from a preceding sound exposure event has some positive or negative influence on the injury risk in a subsequent event. In summary, "combined dose" means the effective dose for the overall composite event, counting the injury in all element events. In contrast, "cumulative dose" refers to the effective dose for one event, counting the injury in only one event, including both the effect of the current event's dose and the left-over effects from preceding events' doses.

To further delineate the difference between "combined dose" and "cumulative dose", let us consider the simple case where all sound exposure events are independent of each other. In this case, the "cumulative dose" is just the dose of the current event since preceding events have no influence on the injury risk of the current event due to independence. On the other hand, the "combined dose" for two identical and independent events is larger than the dose of each event since the total injury risk in the two events is certainly larger than that in each individual event. 
Before we end this section, we examine the connection between the sound exposure energy and the dose combination rule (4). Recall from the definition of SELA (3) that the sound exposure energy of each impulse is

$$
E=E_{0} 10^{\frac{\text { SELA }}{10}}=E_{0} \exp \left(\ln (10) \frac{\text { SELA }}{10}\right)
$$

The sound exposure energy corresponding to the effective combined SELA value (4) can be written out in the same fashion as above

$$
\begin{aligned}
E_{\text {comb }} & =E_{0} \exp \left(\ln (10) \frac{\text { SELA }_{\text {comb }}}{10}\right) \\
& =E_{0} \exp \left(\ln (10) \frac{\mathrm{SELA}+\lambda \log _{10} N}{10}\right) \\
& =E \cdot N^{\frac{\lambda}{10}}
\end{aligned}
$$

where $E$ is the sound exposure energy of each impulse given in (5). For $\lambda=10$, $E_{\text {comb }}$ is simply the sum of individual energies.

$$
E_{\text {comb }}=N \cdot E
$$

For $\lambda<10, E_{\text {comb }}$ is less than the sum of individual energies:

$$
E_{\text {comb }}<N \cdot E
$$

In [6], $\lambda=3.44$ is used for $N \leq 25$ and $\lambda=10$ is used for $N>25$.

\section{Independence or Synergy or Negative Synergy among a Sequence of Doses for Causing Injury}

We study analytically the effects of multiple impulse shots based on the mathematical framework of the logistic dose-response relation and the dose combination rule proposed in [6]. The process of analysis will guide us in finding a self-consistent and reasonable way of extending the dose combination rule from the special case of multiple shots of equal intensity ("homogeneous doses") [6] to the general case of multiple shots of non-uniform intensities ("heterogeneous doses").

We introduce some short notations to facilitate the discussion.

- $S$ : the effective combined SELA of an event where the event may be a single impulse or a composite event consisting of a sequence of heterogeneous events.

- $S_{j}$ : the effective combined SELA of event $j$.

- $G\left\{S_{1}, S_{2}, \cdots, S_{N}\right\}$ : the effective combined SELA for a sequence of $N$ events, respectively with individual SELA values $\left\{S_{1}, S_{2}, \cdots, S_{N}\right\}$. Here each event in the sequence may itself be a composite event consisting of sub-events. Mathematically, function $G\left\{S_{1}, S_{2}, \cdots, S_{N}\right\}$ describes the general dose combination rule, which is yet to be specified.

In [6], the dose combination rule is given for the special case where all events in the sequence are single impulses of the same intensity ("homogeneous doses"). For this special case, function $G\left\{S_{1}, S_{2}, \cdots, S_{N}\right\}$ has the expression 


$$
G \underbrace{\{S, S, \cdots, S\}}_{N \text { impulses }}=S+\lambda \log _{10}(N)
$$

One of the goals in our study is to extend the dose combination rule (9) to the general case where the SELA values $\left\{S_{1}, S_{2}, \cdots, S_{N}\right\}$ are not all equal. In the dose-response relation developed in [6], the dose is fully characterized by the effective combined SELA calculated based on the dose combination rule (9), without distinguishing whether or not the event is a single impulse or a sequence of impulses. This assumption is at least self-consistent.

The self-consistency can be seen by considering a sequence of $2 N$ impulses, each with SELA value $S$. Let us call it view \#1. The sequence can also be viewed as two big events, each big event consisting of $N$ impulses and each big event having the effective combined SELA value $S+\lambda \log _{10}(N)$. Let us call this view $\# 2$. The effective combined SELA value calculated using (9) is the same for both view \#1 and view \#2:

$$
\begin{aligned}
& \text { View \#1: } G \underbrace{\{S, S, \cdots, S\}}_{2 N \text { impulses }}=S+\lambda \log _{10}(2 N) \\
& \text { View \#2: } G\{\underbrace{\{S, S, \cdots, S\}}_{N \text { impulses }}, G \underbrace{\{S, S, \cdots, S\}}_{N \text { impulses }}\} \\
& =G \underbrace{\{S, S, \cdots, S\}}_{N \text { impulses }}+\lambda \log _{10}(2) \\
& =\left(S+\lambda \log _{10}(N)\right)+\lambda \log _{10}(2)=S+\lambda \log _{10}(2 N)
\end{aligned}
$$

For the purpose of extending the formulation to the general case of heterogeneous events, we need to bring events with different doses into a problem that can be solved in the special case of homogeneous events. We consider a sequence of $(m+n)$ impulses of equal intensity, each with SELA value $S$. We group the first $m$ impulses into a composite event $E_{1}$; and group the rest $n$ impulses into a composite event $E_{2}$. Thus, the sequence of $(m+n)$ homogeneous impulses can be viewed as two composite events $E_{1}$ and $E_{2}$ with different effective combined SELA values.

$$
\overbrace{\underbrace{S, S, \cdots, S}_{m \text { impulses }}}^{\text {Event } E_{1}}, \overbrace{\underbrace{S, S, \cdots, S}_{n \text { impulses }}}^{\text {Event } E_{2}}
$$

The effective combined SELA value of event $E_{1}$ is given by (9)

$$
S_{E_{1}}=S+\lambda \log _{10}(m)
$$

The effective combined SELA value of event $E_{2}$ is

$$
S_{E_{2}}=S+\lambda \log _{10}(n)
$$

The effective combined SELA value of events $E_{1}$ and $E_{2}$ is

$$
S_{E_{1}+E_{2}}=S+\lambda \log _{10}(m+n)
$$

With the effective combined SELA values, the injury risks in these composite events are given by the dose-response relation: 


$$
\begin{gathered}
P_{E_{1}}=\frac{1}{1+\exp \left(-\alpha\left(S_{E_{1}}-\mathrm{ID}_{50}\right)\right)} \\
P_{E_{2}}=\frac{1}{1+\exp \left(-\alpha\left(S_{E_{2}}-\mathrm{ID}_{50}\right)\right)} \\
P_{E_{1}+E_{2}}=\frac{1}{1+\exp \left(-\alpha\left(S_{E_{1}+E_{2}}-\mathrm{ID}_{50}\right)\right)}
\end{gathered}
$$

Notice that each injury probability above is directly from the dose-response relation using the SELA value of the event, not including effects from any other events. This is for the case where the event under consideration is treated as a stand-alone event, i.e., not preceded by any other event(s).

While event $E_{1}$ and event $\left(E_{1}+E_{2}\right)$ are indeed stand-alone, event $E_{2}$ is not. Event $E_{2}$ occurs after event $E_{1}$ in the sequence of events $E_{1}$ and $E_{2}$. If events $E_{1}$ and $E_{2}$ are independent of each other, then the three injury probabilities should satisfy the relation

$$
\left(1-P_{E_{1}+E_{2}}\right)-\left(1-P_{E_{1}}\right)\left(1-P_{E_{2}}\right)=0
$$

The independence implies that the probability of no injury in the composite event is the product of no-injury probabilities in individual element events.

If the probability of no injury in the composite event is more than the product of no-injury probabilities in individual element events, $\left(1-P_{E_{1}+E_{2}}\right)-\left(1-P_{E_{1}}\right)\left(1-P_{E_{2}}\right)>0$, then it rules out the independence and indicates some kind of negative synergy in the injury mechanism among individual element events (i.e., immunity passed onto subsequent events). Here immunity means that having experienced the sound exposure but not injured in event $E_{1}$ increases one's conditional probability of escaping injury in the sound exposure of event $E_{2}$, and thus, increases the overall no-injury probability of the composite state $E_{1}+E_{2}$.

Conversely, if the probability of no injury in the composite event is less than the product of no-injury probabilities in individual element events, $\left(1-P_{E_{1}+E_{2}}\right)-\left(1-P_{E_{1}}\right)\left(1-P_{E_{2}}\right)<0$, then it also rules out the independence. In this case, it indicates some kind of positive synergy in the injury mechanism among individual element events (i.e., fatigue damage passed onto subsequent events). Here fatigue damage means that even if the sound exposure (dose) in event $E_{1}$ does not directly cause injury, it nevertheless weakens the subject or otherwise makes the subject more vulnerable so as to increase the subject's conditional injury probability in the sound exposure of event $E_{2}$, and thus decreases the overall no-injury probability of the composite state $E_{1}+E_{2}$.

We examine the sign of $\left(1-P_{E_{1}+E_{2}}\right)-\left(1-P_{E_{1}}\right)\left(1-P_{E_{2}}\right)$ to assess the independence, immunity, or fatigue. First we express $P_{E_{1}}$ as

$$
P_{E_{1}}=\frac{1}{1+\exp \left(-\alpha\left(S_{E_{1}}-\mathrm{ID}_{50}\right)\right)}=\frac{1}{1+\left(10 \frac{S_{E_{1}}-\mathrm{ID}_{50}}{\lambda}\right)^{\frac{-\alpha \lambda}{\ln 10}}} \equiv \frac{1}{1+W_{E_{1}}^{-\eta}}
$$


Parameter $\eta$ and intermediate variable $W_{E_{1}}$ are defined as

$$
\begin{gathered}
\eta \equiv \frac{\alpha \lambda}{\ln 10}, \\
W_{E_{1}}=W\left(S_{E_{1}}\right) \equiv 10^{\frac{S_{E_{1}}-\mathrm{ID}_{50}}{\lambda}}=m \cdot 10^{\frac{S-\mathrm{ID}_{50}}{\lambda}}
\end{gathered}
$$

where we have used the expression of $S_{E_{1}}$ given in (10). In a similar fashion, we can write $P_{E_{2}}$ and $P_{E_{1}+E_{2}}$ as

$$
\begin{gathered}
P_{E_{2}}=\frac{1}{1+W_{E_{2}}^{-\eta}}, W_{E_{2}}=n \cdot 10^{\frac{S-\mathrm{ID}_{50}}{\lambda}} \\
P_{E_{1}+E_{2}}=\frac{1}{1+W_{E_{1}+E_{2}}^{-\eta}}, W_{E_{1}+E_{2}}=(m+n) \cdot 10^{\frac{S-\mathrm{ID}_{50}}{\lambda}}
\end{gathered}
$$

Notice directly from (19), (20) and (21) that quantities $W_{E_{1}}, W_{E_{2}}$ and $W_{E_{1}+E_{2}}$ are related by a simple additive relation

$$
W_{E_{1}+E_{2}}=W_{E_{1}}+W_{E_{2}}
$$

We replace $W_{E_{1}+E_{2}}$ by $W_{E_{1}}+W_{E_{2}}$ and write $\left(1-P_{E_{1}+E_{2}}\right)-\left(1-P_{E_{1}}\right)\left(1-P_{E_{2}}\right)$ as

$$
\begin{aligned}
& \left(1-P_{E_{1}+E_{2}}\right)-\left(1-P_{E_{1}}\right)\left(1-P_{E_{2}}\right) \\
& =\left(1-\frac{1}{1+\left(W_{E_{1}}+W_{E_{2}}\right)^{-\eta}}\right)-\left(1-\frac{1}{1+W_{E_{1}}^{-\eta}}\right)\left(1-\frac{1}{1+W_{E_{2}}^{-\eta}}\right) \\
& =\frac{W_{E_{1}}^{\eta}+W_{E_{2}}^{\eta}-\left(W_{E_{1}}+W_{E_{2}}\right)^{\eta}+\left(W_{E_{1}} W_{E_{2}}\right)^{\eta}}{\left(1+\left(W_{E_{1}}+W_{E_{2}}\right)^{\eta}\right)\left(1+W_{E_{1}}^{\eta}\right)\left(1+W_{E_{2}}^{\eta}\right)}
\end{aligned}
$$

Since the denominator is always positive, it follows that the numerator determines the sign of $\left(1-P_{E_{1}+E_{2}}\right)-\left(1-P_{E_{1}}\right)\left(1-P_{E_{2}}\right)$. We introduce a theorem.

Theorem 1: When $\eta<1$, we always have

$$
W_{E_{1}}^{\eta}+W_{E_{2}}^{\eta}-\left(W_{E_{1}}+W_{E_{2}}\right)^{\eta}+\left(W_{E_{1}} W_{E_{2}}\right)^{\eta}>0
$$

for all $W_{E_{1}}>0$ and $W_{E_{2}}>0$.

The proof of the theorem is presented in Appendix. From Theorem 1, we see that when the parameter $\eta \equiv \frac{\alpha \lambda}{\ln 10}$ is less than 1 , the quantity $\left(1-P_{E_{1}+E_{2}}\right)-\left(1-P_{E_{1}}\right)\left(1-P_{E_{2}}\right)$ is always positive, which clearly rules out the independence and indicates negative synergy (immunity) in the injury mechanism between preceding and subsequent events. In [6], the logistic dose-response relation based on experimental data has $\alpha=0.1$ and $\lambda=3.44$ or 10 . For these parameter values, the quantity $\eta \equiv \frac{\alpha \lambda}{\ln 10}$ is 0.15 or 0.34 , well within the range of $\eta<1$. Therefore, in the framework of the logistic doseresponse relation and the dose combination rule [6], we draw two conclusions:

1) a subsequent event is not independent of the presence of preceding 
event(s), and

2) the effect of a preceding event on the subsequent events is manifested in the form of immunity instead of fatigue; that is, a sequence of sound exposure events demonstrates negative synergy in causing injury.

To further distinguish the dose combination rule (4) from the case of independent events, we consider a sequence of $N$ impulse shots, each with SELA value $S$. We look at the difference between these two cases in the probability of no injury as a function of $N$. For each individual shot, when viewed as a stand-alone event, the probability of no injury is given by the dose-response relation (1):

$$
1-P(S)=\frac{1}{\mathrm{e}^{\alpha\left(S-\mathrm{ID}_{50}\right)}+1}
$$

In the case of independent events, the probability of no injury for the whole sequence is

$$
(1-P(S))^{N}=\left(\frac{1}{\mathrm{e}^{\alpha\left(S-\mathrm{ID}_{50}\right)}+1}\right)^{N}
$$

When we use the dose combination rule (4) to combine the $N$ impulse shots into one effective SELA value $S_{\text {comb }}=S+\lambda \log _{10}(N)$, the probability of no injury has the expression

$$
1-P\left(S_{\text {comb }}\right)=\frac{1}{\mathrm{e}^{\alpha\left(S_{\text {comb }}-\mathrm{ID}_{50}\right)}+1}=\frac{1}{N^{\eta} \cdot \mathrm{e}^{\alpha\left(S-\mathrm{ID}_{50}\right)}+1}
$$

where $\eta$ is given in (18). Figure 2 displays the plots of no-injury probability vs $N$ for these two cases, which clearly demonstrates the difference. In the case of independent events, the no-injury probability decays exponentially with respect

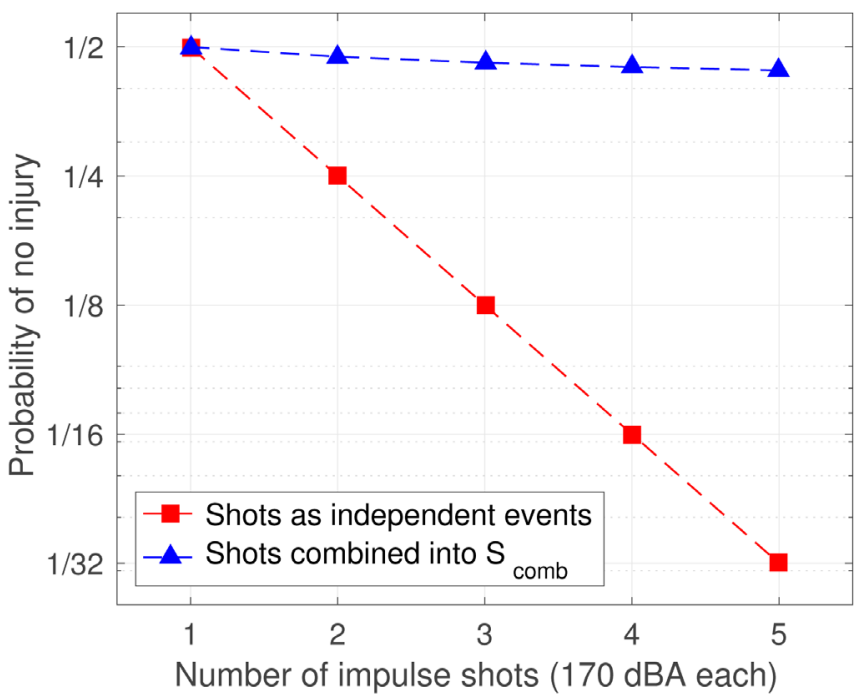

Figure 2. Comparison of the dose combination rule in [6] and the case of independent events. Plots of no-injury probability vs $N$ for a sequence of $N$ impulse shots, each with SELA $=170 \mathrm{dBA}$. The probability is shown in the logarithmic scale. The parameters used are $\alpha=0.1, \mathrm{ID}_{50}=170 \mathrm{dBA}, \lambda=3.44$ from [6]. 
to the number of shots $N$. In the logarithmic scale, the exponential decay yields a downward constant slope. In sharp contrast, the no-injury probability corresponding to the dose combination rule in [6] has a much slower decrease with respect to $N$.

\section{Dose Combination Rules}

Building on the insight gained in the analysis above, we extend the dose combination rule for a homogeneous sequence of impulse shots [6], to the general case of $N$ sound exposure events of non-uniform SELA values where each event in the sequence may be a composite event consisting of sub-events. Then we construct the dose combination rule for the case of independent events and compare the two rules.

\subsection{Extension of the Dose Combination Rule to the General Case of Multiple Heterogeneous Doses}

In Equations (17), (20), and (21) above, we wrote the dose-response relation in terms of an intermediate variable $W(S)$. When we divide a long sequence of impulses of the same intensity into two segments and treat each segment as a composite event, the two segments and the whole sequence are each described by its effective combined SELA value $\left(S_{E_{1}}, S_{E_{2}}, S_{E_{1}+E_{2}}\right)$. In particular, the injury probability of each segment is described by the dose-response relation with the effective combined SELA value (when it is treated as a stand-alone composite event, excluding the effects of sound exposure in preceding events). While it is not directly obvious how we can combine $S_{E_{1}}$ and $S_{E_{2}}$ to obtain $S_{E_{1}+E_{2}}$, in the analysis above a key observation is that the intermediate variable $W(S)$ is additive:

$$
W\left(S_{E_{1}+E_{2}}\right)=W\left(S_{E_{1}}\right)+W\left(S_{E_{2}}\right)
$$

Thus, we propose to extend the dose combination rule by summing the intermediate variable $W(S)$ over all events in the sequence. More specifically, consider a sequence of $N$ sound exposure events where each event in the sequence may be a composite event consisting of sub-events. Each sound exposure event in the sequence is described by its effective combined SELA value. The whole sequence is specified by SELA values of the $N$ events: $\left\{S_{j}, j=1,2, \cdots, N\right\}$. When we view the sequence as an overall composite event, the effective combined SELA value for the whole sequence is denoted by $G\left\{S_{1}, S_{2}, \cdots, S_{N}\right\}$. Mathematically, function $G\left\{S_{1}, S_{2}, \cdots, S_{N}\right\}$ is specified via the corresponding intermediate variable $W\left(G\left\{S_{1}, S_{2}, \cdots, S_{N}\right\}\right) \equiv 10 \frac{G\left\{S_{1}, S_{2}, \cdots, S_{N}\right\}-\mathrm{ID}_{50}}{\lambda}$, which is given as the sum of $W\left(S_{j}\right)$ over individual events in the sequence.

$$
W\left(G\left\{S_{1}, S_{2}, \cdots, S_{N}\right\}\right)=\sum_{j=1}^{N} W\left(S_{j}\right)
$$

This relation on the intermediate variable $W$ leads to the generalized dose 
combination rule:

$$
G\left\{S_{1}, S_{2}, \cdots, S_{N}\right\}=\lambda \log _{10}\left(\sum_{j=1}^{N} 10^{\frac{S_{j}-\mathrm{ID}_{50}}{\lambda}}\right)+\mathrm{ID}_{50}
$$

Note that when all $S_{j}$ 's are equal to $S$, (29) is reduced to

$$
G\{S, S, \cdots, S\}=S+\lambda \log _{10} N,
$$

Therefore, the generalized dose combination rule (29) is consistent with the special case dose combination rule described in [6].

The generalized dose combination rule, as given in (29), appears to be affected by the median injury dose $\mathrm{ID}_{50}$. Actually it is not affected by $\mathrm{ID}_{50}$. We can get rid of $\mathrm{ID}_{50}$ from (29). Let $S_{\max } \equiv \max _{k} S_{k}$. We re-write the generalized dose combination rule as

$$
G\left\{S_{1}, S_{2}, \cdots, S_{N}\right\}=S_{\max }+\lambda \log _{10}\left(\sum_{j=1}^{N} 10^{\frac{S_{j}-S_{\max }}{\lambda}}\right),
$$

where $S_{\max } \equiv \max _{k} S_{k}$.

Let us look at a few examples of combining two sound exposure events.

$$
\begin{aligned}
G\{S, S\}=S & +\lambda \log _{10}(2)=S+0.3 \lambda(\mathrm{dB}) \\
G\{S,(S+3)\} & =(S+3)+\lambda \log _{10}\left(1+10^{\frac{-3}{\lambda}}\right) \\
& = \begin{cases}(S+3)+0.19, & \lambda=3.44 \\
(S+3)+1.76, & \lambda=10\end{cases}
\end{aligned}
$$

The effective combined SELA of two events with $\mathrm{S}$ and $(S+3) \mathrm{dB}$ is $0.19 \mathrm{~dB}$ and $1.76 \mathrm{~dB}$ above the larger one, respectively for $\lambda=3.44$ and $\lambda=10$. In terms of SELA values, $3 \mathrm{~dB}$ difference means a factor of 2 in sound exposure energy.

$$
\begin{aligned}
G\{S,(S+10)\} & =(S+10)+\lambda \log _{10}\left(1+10^{\frac{-10}{\lambda}}\right) \\
& = \begin{cases}(S+10)+0.002, & \lambda=3.44 \\
(S+10)+0.41, & \lambda=10\end{cases}
\end{aligned}
$$

The effective combined SELA of two events with $S$ and $(S+10) \mathrm{dB}$ is $0.002 \mathrm{~dB}$ and $0.41 \mathrm{~dB}$ above the larger one, respectively for $\lambda=3.44$ and $\lambda=10$. In terms of SELA values, $10 \mathrm{~dB}$ difference means a factor of 10 in sound exposure energy. In general, for $\Delta S \geq \lambda$, the effective combined SELA of two events with $S$ and $S+\Delta S$ is approximately

$$
\begin{aligned}
G\{S,(S+\Delta S)\} & =(S+\Delta S)+\lambda \log _{10}\left(1+10^{\frac{-\Delta S}{\lambda}}\right) \\
& \approx(S+\Delta S)+\frac{\lambda}{\ln (10)} 10^{\frac{-\Delta S}{\lambda}}
\end{aligned}
$$


For moderately large $\Delta S / \lambda$, the combined SELA is just the larger one of the two event.

The generalized dose combination rule (31) is an extension of the rule from [6], which is based on empirical data. For that reason, and for distinguishing different rules of combining doses, we shall refer to (31) as the extended empirical dose combination rule (or just simply, empirical rule), and specifically denote it as $G_{\text {Empr }}\left\{S_{1}, S_{2}, \cdots, S_{N}\right\}$ when necessary. This will set up the proper notation framework allowing us to discuss the dose combination rule for independent events and compare it with the empirical rule, which is the subject of the next subsection.

\subsection{Dose Combination Rule for the Case of Independent Events and Comparison with the Empirical Rule}

We study the hypothetical situation where all sound exposure events are independent of each other. We want to write out and examine the dose combination rule corresponding to this situation. Consider a sequence of $N$ sound exposure events $S E Q=\left\{E_{j}, j=1,2, \cdots, N\right\}$ with individual SELA values $\left\{S_{j}, j=1,2, \cdots, N\right\}$. The probability of no injury in each individual event is calculated from the dose-response relation (1):

$$
1-P_{E_{j}}=1-\frac{1}{1+\exp \left(-\alpha\left(S-\mathrm{ID}_{50}\right)\right)}=\frac{1}{\exp \left(\alpha\left(S-\mathrm{ID}_{50}\right)\right)+1}
$$

When all events are independent of each other, the probability of no injury for the whole sequence is the product of no-injury probabilities of individual events

$$
1-P_{S E Q}=\prod_{j=1}^{N}\left(1-P_{E_{j}}\right)=\frac{1}{\prod_{j=1}^{N}\left(\exp \left(\alpha\left(S_{j}-\mathrm{ID}_{50}\right)\right)+1\right)}
$$

On the other hand, the probability of no injury for the whole sequence is governed by the dose-response relation with the effective combined dose denoted by $G_{\text {Indp }}\left\{S_{1}, S_{2}, \cdots, S_{N}\right\}$, which is what we want to find. Here we use the subscript "Indp" to empasize that the function refers to the case of independent events.

$$
1-P_{S E Q}=\frac{1}{\exp \left(\alpha\left(G_{\text {Indp }}\left\{S_{1}, S_{2}, \cdots, S_{N}\right\}-\mathrm{ID}_{50}\right)\right)+1}
$$

Mathematically, function $G_{\text {Indp }}\left\{S_{1}, S_{2}, \cdots, S_{N}\right\}$ is defined by equating (32) and (33).

$$
\exp \left(\alpha\left(G_{\text {Indp }}\left\{S_{1}, S_{2}, \cdots, S_{N}\right\}-\mathrm{ID}_{50}\right)\right)=\prod_{j=1}^{N}\left(\exp \left(\alpha\left(S_{j}-\mathrm{ID}_{50}\right)\right)+1\right)-1
$$

Solving for $G_{\text {Indp }}\left\{S_{1}, S_{2}, \cdots, S_{N}\right\}$, we arrive at:

$$
G_{\text {Indp }}\left\{S_{1}, S_{2}, \cdots, S_{N}\right\}=\frac{1}{\alpha} \ln \left(\prod_{j=1}^{N}\left(\exp \left(\alpha\left(S_{j}-\mathrm{ID}_{50}\right)\right)+1\right)-1\right)+\mathrm{ID}_{50}
$$


(35) is the dose-combination rule for the case of independent events. We compare (35) to the empirical rule (31). We first look at the dependence on $N$ for a homogeneous sequence of $N$ events, each with SELA value $S$. The empirical rule is a linear function of $\log _{10}(N)$.

$$
G_{\text {Empr }}\{S, S, \cdots, S\}=S+\lambda \log _{10}(N)
$$

For large $N$, the combined dose of independent events asymptotically behaves like

$$
G_{\text {Indp }}\{S, S, \cdots, S\} \approx N \cdot \frac{1}{\alpha} \ln \left(\mathrm{e}^{\alpha\left(S-\mathrm{ID}_{50}\right)}+1\right)+\mathrm{ID}_{50}
$$

Figure 3 compares the dependence on $N$ of these two dose combination rules.

Unlike the empirical rule (31), the combined dose of independent events (35) does depend on the median injury dose $\mathrm{ID}_{50}$. To see the dependence on $\mathrm{ID}_{50}$, we examine the combined dose of two independent events $(N=2)$, each with SELA value $S$.

$$
\begin{aligned}
& G_{\text {Indp }}\{S, S\}=S+\frac{1}{\alpha} \ln \left(\mathrm{e}^{\alpha\left(S-\mathrm{ID}_{50}\right)}+2\right) \\
& \approx \begin{cases}S+\frac{1}{\alpha} \ln (2), & \alpha\left(S-\mathrm{ID}_{50}\right) \text { moderately large, negative } \\
S+\frac{1}{\alpha} \ln (3), & S=\mathrm{ID}_{50} \\
2\left(S-\mathrm{ID}_{50}\right)+\mathrm{ID}_{50}, & \alpha\left(S-\mathrm{ID}_{50}\right) \text { moderately large, positive }\end{cases}
\end{aligned}
$$

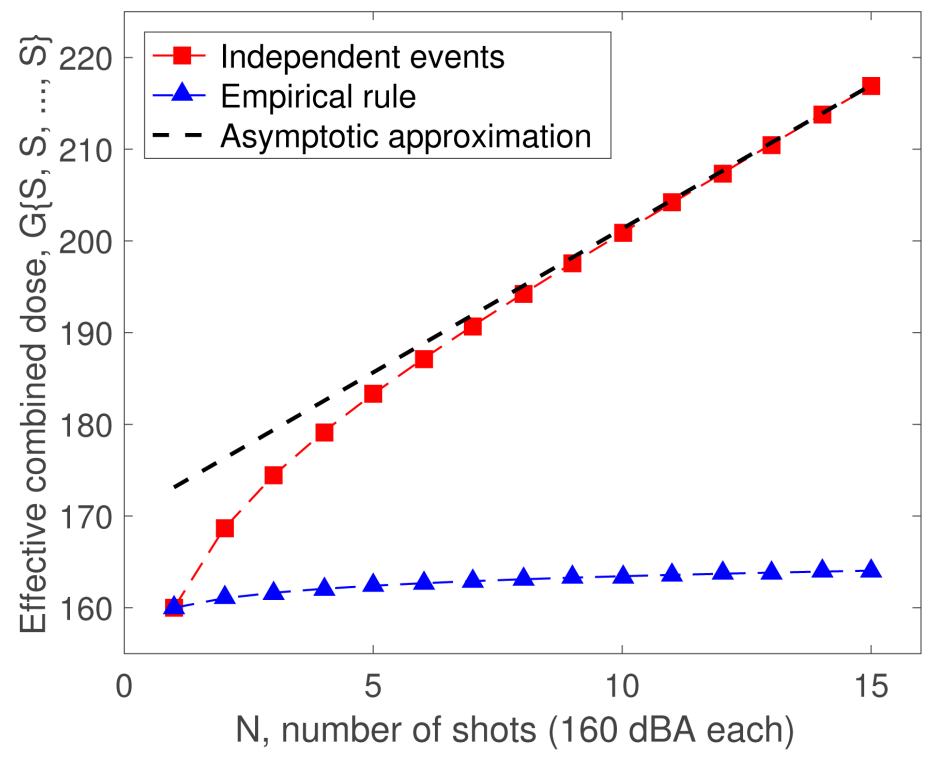

Figure 3. Comparison of the two dose combination rules, $G_{\text {Indp }}$ and $G_{\text {Empr }}$ for a sequence of $N$ impulse shots, each with SELA = $160 \mathrm{dBA}$. Plots of the effective combined dose vs $N$, respectively, for the two cases, along with the asymptotic approximation for independent events. For large $N$, the combined dose of independent events asymptotically approaches a linear function of $N$ while the empirical rule is a linear function of $\log _{10}(N)$. 
For $S$ moderately below the median injury dose $\operatorname{ID}_{50}$, the combined dose of independent events behaves the same way as the empirical rule with the increment for empirical rule $\lambda \log _{10}(2)$ replaced by $\frac{1}{\alpha} \ln (2)$. Recall that parameters $\alpha$ and $\lambda$ are related by parameter $\eta$ defined in (18): $\eta=\frac{\alpha \lambda}{\ln (10)}$. As a result, the two increments are related by

$$
\frac{1}{\alpha} \ln (2)=\frac{1}{\eta} \times \lambda \log _{10}(2)
$$

Thus, for $\eta$ significantly below 1 , the increment for independent events is significantly higher than that in the empirical rule. For example, for $\eta=0.15$, the two increments differ by a factor of 6.67 .

For $S$ moderately above the median injury dose $\mathrm{ID}_{50}$, however, the combined dose for independent events behaves very differently from the empirical rule. Instead of adding a fixed increment of $\lambda \log _{10}(2)$, the combined dose for independent events doubles the amount of $S$ above the median injury dose $\mathrm{ID}_{50}$.

To illustrate the behaviors of $G_{\text {Indp }}\{S, S\}$ described in (36), we plot $\left(G_{\text {Indp }}\{S, S\}-S\right)$, the resulting increment in combining two independent events of equal SELA values $(S)$. Figure 4 shows $\left(G_{\text {Indp }}\{S, S\}-S\right)$ vs $S$ and its asymptotic approximations respectively for low $S$ and high $S$, along with $\left(G_{\text {Empr }}\{S, S\}-S\right)$ vs $S$, the corresponding plot for the empirical rule. The median injury dose $\mathrm{ID}_{50}$ is indicated by the dotted gray vertical line in Figure 4 . Notice that for $S$ above $\operatorname{ID}_{50}$, the combined dose $G_{\text {Indp }}\{S, S\}$ is significantly

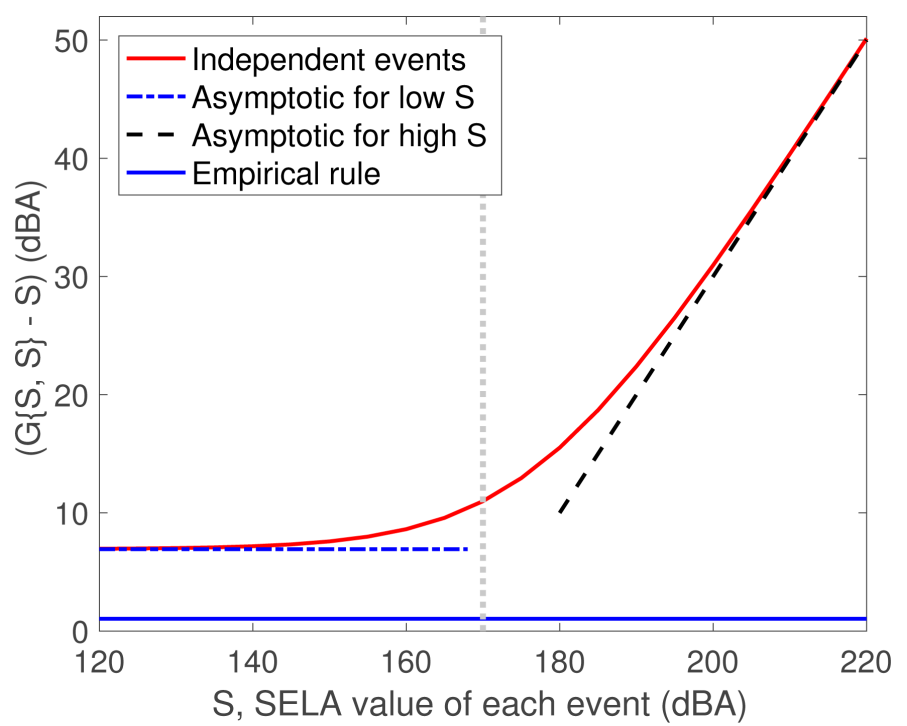

Figure 4. Comparison of the two dose combination rules: $G_{\text {Indp }}\{S, S\}$ and $G_{\text {Empr }}\{S, S\}$. Plot of $\left(G_{\text {Indp }}\{S, S\}-S\right)$ vs $S$, its asymptotic approximations respectively for low $S$ and high $S$, and plot of $\left(G_{\text {Empr }}\{S, S\}-S\right)$ vs $S$. The parameters used are $\lambda=3.44, \alpha=0.1$, $\mathrm{ID}_{50}=170 \mathrm{dBA}$ from [6]. The gray dotted vertical line indicates the median injury dose. 
higher than $S$ and the increment $\left(G_{\text {Indp }}\{S, S\}-S\right)$ is approximately $\left(S-\operatorname{ID}_{50}\right)$. For $S$ below $\operatorname{ID}_{50}$, the increment $\left(G_{\text {Indp }}\{S, S\}-S\right)$ is approximately constant with respect to $S$ but is much higher than that for the empirical rule.

To demonstrate the dependence on the median injury dose $\mathrm{ID}_{50}$, in Figure 5, we plot the combined dose of two events with equal SELA value $S=160(\mathrm{dBA})$, as a function of $\mathrm{ID}_{50}$, respectively for the two combination rules. The combined dose of independent events is a decreasing function of $\mathrm{ID}_{50}$; the empirical rule has no dependence on $\mathrm{ID}_{50}$.

\section{Behaviors of Immunity in the Extended Empirical Dose-Response Formulation}

We start this section by summarizing the extended empirical dose-response formulation for multiple sound exposure events with heterogeneous SELA values $\left\{S_{1}, S_{2}, \cdots, S_{N}\right\}$. The formulation contains two components: 1) the rule for combining the heterogeneous doses $\left\{S_{1}, S_{2}, \cdots, S_{N}\right\}$ into one effective dose $S_{\text {comb }}$ and 2) the dose-response relation mapping the effective dose $S_{\text {comb }}$ to the occurrence probability of injury.

- The extended empirical dose combination rule:

$$
S_{\text {comb }}=S_{\text {max }}+\lambda \log _{10}\left(\sum_{j=1}^{N} 10^{\frac{S_{j}-S_{\max }}{\lambda}}\right), \quad S_{\max } \equiv \max _{k} S_{k}
$$

- The dose-response relation:

$$
P\left(S_{\text {comb }}\right)=\frac{1}{1+\exp \left(-\alpha\left(S_{\text {comb }}-\mathrm{ID}_{50}\right)\right)}
$$

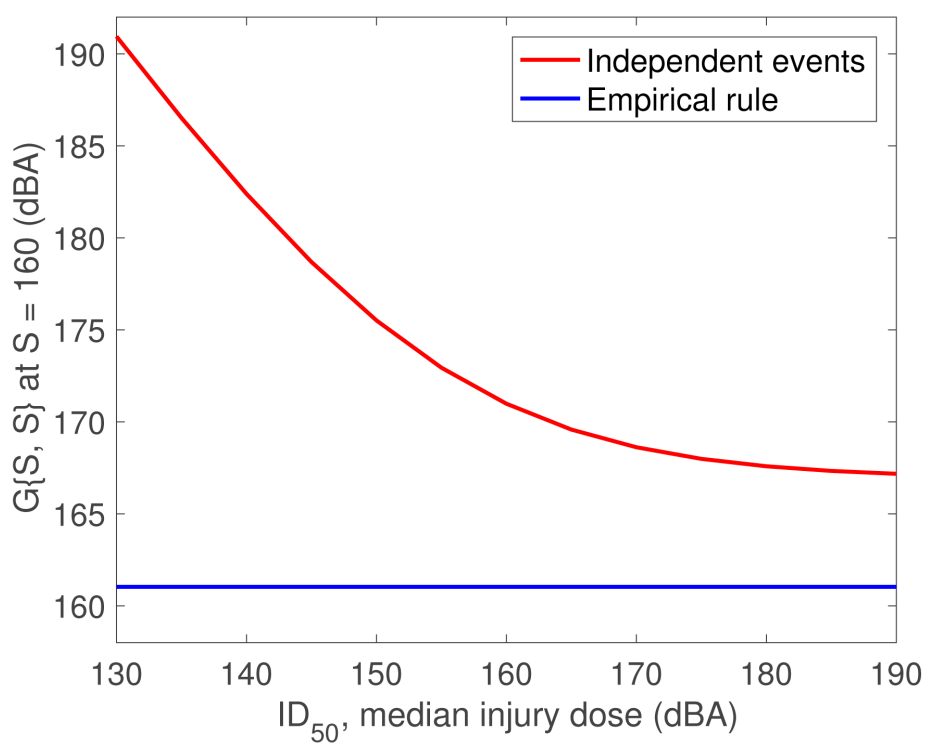

Figure 5. Comparison of the two dose combination rules: $G_{\text {Indp }}\{S, S\}$ and $G_{\text {Empr }}\{S, S\}$. Plot of $G\{S, S\}$ at $S=160$ as a function of the median injury dose $\mathrm{ID}_{50}$. The parameters used are $\lambda=3.44, \alpha=0.1$ from [6]. 
In this section, we study the immunity in the dose-response formulation (37) and (38). We consider the case of two composite sound exposure events $E_{1}$ and $E_{2}$, respectively with effective combined SELA values $S_{E_{1}}$ and $S_{E_{2}}$. In the previous section, we wrote the dose-response relation (38) in terms of intermediate variable $W(S) \equiv 10^{\frac{S-\mathrm{ID}_{50}}{\lambda}}$ and parameter $\eta \equiv \frac{\alpha \lambda}{\ln (10)}$.

$$
P(S)=\frac{1}{1+W(S)^{-\eta}}, \quad W(S) \equiv 10^{\frac{S-\mathrm{ID}_{50}}{\lambda}}, \quad \eta \equiv \frac{\alpha \lambda}{\ln (10)}
$$

We examine several probabilities via intermediate variable $W(S)$.

$$
\begin{gathered}
\operatorname{Pr}\left(\text { no injury in } E_{1}\right)=1-P\left(S_{E_{1}}\right)=\frac{1}{1+W\left(S_{E_{1}}\right)^{\eta}}=\frac{1}{1+W_{1}^{\eta}}, \quad W \\
\operatorname{Pr}\left(\text { no injury in } E_{1} \text { and no injury in } E_{2}\right) \\
=1-P\left(S_{E_{1}+E_{2}}\right)=\frac{1}{1+\left(W_{1}+W_{2}\right)^{\eta}}, \quad W_{2} \equiv W\left(S_{E_{2}}\right) \\
\\
\operatorname{Pr}\left(\text { no injury in } E_{2} \mid \text { no injury in } E_{1}\right) \\
=\frac{\operatorname{Pr}\left(\text { no injury in } E_{1} \text { and no injury in } E_{2}\right)}{\operatorname{Pr}\left(\text { no injury in } E_{1}\right)} \\
=\frac{1-P\left(S_{E_{1}+E_{2}}\right)}{1-P\left(S_{E_{1}}\right)}=\frac{1+W_{1}^{\eta}}{1+\left(W_{1}+W_{2}\right)^{\eta}}
\end{gathered}
$$

When we treat $E_{2}$ as a stand-alone event (i.e. excluding any effect left over from the sound exposure in the preceding event $E_{1}$ ), the probability of no injury in event $E_{2}$ is

$$
\begin{aligned}
& \operatorname{Pr}\left(\text { no injury in } E_{2} \text {, excluding the effect of } E_{1}\right) \\
& =1-P\left(S_{E_{2}}\right)=\frac{1}{1+W_{2}^{\eta}}
\end{aligned}
$$

From Theorem 1 in Section 3, we have

$$
W_{1}^{\eta}+W_{2}^{\eta}+\left(W_{1} W_{2}\right)^{\eta}>\left(W_{1}+W_{2}\right)^{\eta}
$$

Adding 1 to each side of the inequality and then factoring the left-hand side, we obtain

$$
\left(1+W_{1}^{\eta}\right)\left(1+W_{2}^{\eta}\right)>1+\left(W_{1}+W_{2}\right)^{\eta}
$$

which yields

$$
\frac{1+W_{1}^{\eta}}{1+\left(W_{1}+W_{2}\right)^{\eta}}>\frac{1}{1+W_{2}^{\eta}}
$$

Comparing (40) and (41) gives us

$$
\begin{aligned}
& \operatorname{Pr}\left(\text { no injury in } E_{2} \mid \text { no injury in } E_{1}\right) \\
& >\operatorname{Pr}\left(\text { no injury in } E_{2} \text {, excluding the effect of } E_{1}\right)
\end{aligned}
$$


Therefore, we conclude that the effect of sound exposure in $E_{1}$ provides an immunity in the subsequent event $E_{2}$. We assess the effect of immunity quantitatively as a percentage of decrease in probability of injury in event $E_{2}$ due to the preceding event $E_{1}$, and call it the immunity factor of event $E_{1}$ on event $E_{2}$. Mathematically, the immunity factor is defined as

$$
\phi\left(S_{E_{1}}, S_{E_{2}}\right) \equiv 1-\frac{\operatorname{Pr}\left(\text { injury in } E_{2} \mid \text { no injury in } E_{1}\right)}{\operatorname{Pr}\left(\text { injury in } E_{2} \text {, excluding the effect of } E_{1}\right)}
$$

In terms of immunity factor $\phi$, the conditional probability of injury given the prior sound exposure and the unconditional probability of injury are related by

$$
\begin{aligned}
& \operatorname{Pr}\left(\text { injury in } E_{2} \mid \text { no injury in } E_{1}\right) \\
& =(1-\phi) \times \operatorname{Pr}\left(\text { injury in } E_{2} \text {, excluding the effect of } E_{1}\right)
\end{aligned}
$$

Below we study the behavior of immunity factor in four regimes of doses.

We write probabilities in terms of intermediate variable $W(S)$, and express the immunity factor as a function of $W_{1}$ and $W_{2}$ :

$$
\begin{aligned}
\phi\left(S_{E_{1}}, S_{E_{2}}\right) & =\frac{\left(1-\frac{1}{1+W_{2}^{\eta}}\right)-\left(1-\frac{1+W_{1}^{\eta}}{1+\left(W_{1}+W_{2}\right)^{\eta}}\right)}{1-\frac{1}{1+W_{2}^{\eta}}} \\
& =\frac{W_{1}^{\eta}+W_{2}^{\eta}-\left(W_{1}+W_{2}\right)^{\eta}+\left(W_{1} W_{2}\right)^{\eta}}{W_{2}^{\eta}\left[1+\left(W_{1}+W_{2}\right)^{\eta}\right]}
\end{aligned}
$$

We consider regimes described in terms of intermediate variable $W(S) \equiv 10^{\frac{S-\mathrm{ID}_{50}}{\lambda}}$.

- $W=O(1)$ :

The corresponding SELA value has the range $S=\mathrm{ID}_{50}+\lambda \log _{10}(O(1))$ which is a small neighborhood around $S=\mathrm{ID}_{50}$

- $W=O(\epsilon)$ :

The corresponding SELA value has the range $S=\mathrm{ID}_{50}-\lambda \log _{10}(O(1 / \epsilon))$ which is moderately below the median injury dose $\mathrm{ID}_{50}$.

- $W=O(1 / \epsilon)$ :

The corresponding SELA value has the range $S=\mathrm{ID}_{50}-\lambda \log _{10}(O(1 / \epsilon))$ which is moderately above the median injury dose ID $_{50}$

In the analysis below, we assume $\eta \equiv \frac{\alpha \lambda}{\ln (10)}<1$. In particular, we will look at the case of $\eta=0.15$, corresponding to $\alpha=0.1$ and $\lambda=3.44$, the parameter values from [6].

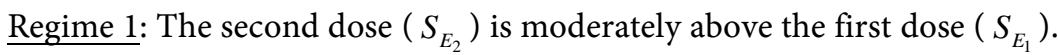

$$
S_{E_{2}}-S_{E_{1}}=\lambda \log _{10}(O(1 / \epsilon))
$$

which in turn yields

$$
\frac{W_{1}}{W_{2}}=10^{\frac{S_{E_{1}}-S_{E_{2}}}{\lambda}}=O(\epsilon)=\text { small }
$$


We use the small parameter $\frac{W_{1}}{W_{2}}=O(\epsilon)$ to derive an asymptotic expression for the immunity factor $\phi$. In the expression of $\phi$ given in (48), dividing both the numerator and the denominator by $W_{2}^{\eta}$, we write $\phi$ as a function of $\frac{W_{1}}{W_{2}}$ and $W_{2}$ as follows:

$$
\phi\left(S_{E_{1}}, S_{E_{2}}\right)=\frac{\left(\frac{W_{1}}{W_{2}}\right)^{\eta}\left(1+W_{2}^{\eta}\right)+1-\left(1+\frac{W_{1}}{W_{2}}\right)^{\eta}}{1+W_{2}^{\eta}\left(1+\frac{W_{1}}{W_{2}}\right)^{\eta}}
$$

Using the Taylor expansion $\left(1+\frac{W_{1}}{W_{2}}\right)^{\eta}=1+\eta \frac{W_{1}}{W_{2}}+\cdots$, and noticing that for $\eta<1$, we have $\frac{W_{1}}{W_{2}}=O(\epsilon) \ll O\left(\epsilon^{\eta}\right)=\left(\frac{W_{1}}{W_{2}}\right)^{\eta}$, we obtain

$$
\begin{aligned}
& \phi\left(S_{E_{1}}, S_{E_{2}}\right)=\frac{\left(\frac{W_{1}}{W_{2}}\right)^{\eta}\left(1+W_{2}^{\eta}\right)+1-\left(1+\eta \frac{W_{1}}{W_{2}}+\cdots\right)}{1+W_{2}^{\eta}\left(1+\eta \frac{W_{1}}{W_{2}}+\cdots\right)} \\
& =\frac{\left(\frac{W_{1}}{W_{2}}\right)^{\eta}\left(1+W_{2}^{\eta}\right)+\cdots}{1+W_{2}^{\eta}+\cdots}=\left(\frac{W_{1}}{W_{2}}\right)^{\eta}+\cdots \\
& =10^{\frac{S_{E_{1}}-S_{E_{2}}}{\lambda} \eta}+\cdots=O\left(\epsilon^{\eta}\right)=\text { small }
\end{aligned}
$$

Conclusion for Regime 1: The immunity caused by the preceding dose on the subsequent dose of moderately higher SELA value, is small.

Regime 2: The second dose $\left(S_{E_{2}}\right)$ is moderately below the first dose $\left(S_{E_{1}}\right)$.

$$
S_{E_{2}}-S_{E_{1}}=-\lambda \log _{10}(O(1 / \epsilon))
$$

which leads to

$$
\frac{W_{2}}{W_{1}}=10^{\frac{S_{E_{2}}-S_{E_{1}}}{\lambda}}=O(\epsilon)=\text { small }
$$

We use the small parameter $\frac{W_{2}}{W_{1}}=O(\epsilon)$ to derive an asymptotic expression for the immunity factor $\phi$. Dividing both the numerator and the denominator by $W_{1}^{\eta}$ in the formula of $\phi$ in Equation (48), we express $\phi$ in terms of $\frac{W_{2}}{W_{1}}$ and $W_{1}$ :

$$
\phi\left(S_{E_{1}}, S_{E_{2}}\right)=\frac{\left(\frac{W_{2}}{W_{1}}\right)^{\eta}\left(1+W_{1}^{\eta}\right)+1-\left(1+\frac{W_{2}}{W_{1}}\right)^{\eta}}{\left(\frac{W_{2}}{W_{1}}\right)^{\eta}\left[1+W_{1}^{\eta}\left(1+\frac{W_{2}}{W_{1}}\right)^{\eta}\right]}
$$


Substituting in the Taylor expansions $\left(1+\frac{W_{2}}{W_{1}}\right)^{\eta}=1+\eta \frac{W_{2}}{W_{1}}+O\left(\epsilon^{2}\right)$, and dividing the numerator and the denominator by $\left(\frac{W_{2}}{W_{1}}\right)^{\eta}\left(1+W_{1}^{\eta}\right)$, we get

$$
\begin{aligned}
\phi\left(S_{E_{1}}, S_{E_{2}}\right) & =\frac{\left(\frac{W_{2}}{W_{1}}\right)^{\eta}\left(1+W_{1}^{\eta}\right)-\eta \frac{W_{2}}{W_{1}}+O\left(\epsilon^{2}\right)}{\left(\frac{W_{2}}{W_{1}}\right)^{\eta}\left[\left(1+W_{1}^{\eta}\right)+W_{1}^{\eta}\left(\eta \frac{W_{2}}{W_{1}}+O\left(\epsilon^{2}\right)\right)\right]} \\
& =\frac{1-\frac{\eta}{1+W_{1}^{\eta}}\left(\frac{W_{2}}{W_{1}}\right)^{1-\eta}+\frac{O\left(\epsilon^{2-\eta}\right)}{1+W_{1}^{\eta}}}{1+\frac{W_{1}^{\eta}}{1+W_{1}^{\eta}}\left(\eta \frac{W_{2}}{W_{1}}+O\left(\epsilon^{2}\right)\right)}
\end{aligned}
$$

Applying the expansion $\left(\frac{1}{1+z}\right)=(1-z+\cdots)$ to the denominator, we arrive at

$$
\begin{aligned}
\phi\left(S_{E_{1}}, S_{E_{2}}\right) & =1-\frac{\eta}{1+W_{1}^{\eta}}\left(\frac{W_{2}}{W_{1}}\right)^{1-\eta}-\eta \frac{W_{1}^{\eta}}{1+W_{1}^{\eta}} \frac{W_{2}}{W_{1}}+\cdots \\
& =1-\eta\left(\frac{W_{2}}{W_{1}}\right)^{1-\eta}\left(\frac{1+W_{2}^{\eta}}{1+W_{1}^{\eta}}\right)+\cdots
\end{aligned}
$$

When $S_{E_{1}}$ and $S_{E_{2}}$ move together from low to high with the difference $\left(S_{E_{2}}-S_{E_{1}}\right)$ unchanged, the pair $\left(W_{1}, W_{2}\right)$ increases in magnitude from small to large with the ratio $\frac{W_{2}}{W_{1}}=10^{\frac{S_{E_{2}}-S_{E_{1}}}{\lambda}}$ fixed. As a result, the factor $\left(\frac{1+W_{2}^{\eta}}{1+W_{1}^{\eta}}\right)$ varies from 1 at low $\left(S_{E_{1}}, S_{E_{2}}\right)$ to $\left(\frac{W_{2}}{W_{1}}\right)^{\eta}=O\left(\epsilon^{\eta}\right)$ at high $\left(S_{E_{1}}, S_{E_{2}}\right)$.

$$
\frac{1+W_{2}^{\eta}}{1+W_{1}^{\eta}} \approx \begin{cases}1, & \left(S_{E_{1}}, S_{E_{2}}\right) \text { moderately below } \mathrm{ID}_{50} \\ \left(\frac{W_{2}}{W_{1}}\right)^{\eta}, & \left(S_{E_{1}}, S_{E_{2}}\right) \text { moderately above } \mathrm{ID}_{50}\end{cases}
$$

With the result of (53), we write the immunity factor as

$$
\phi\left(S_{E_{1}}, S_{E_{2}}\right) \approx \begin{cases}1-\eta 10^{\frac{S_{E_{2}}-S_{E_{1}}}{\lambda}(1-\eta)}, & \left(S_{E_{1}}, S_{E_{2}}\right) \text { moderately below } \mathrm{ID}_{50} \\ 1-\eta 10^{\frac{S_{E_{2}}-S_{E_{1}}}{\lambda}}, & \left(S_{E_{1}}, S_{E_{2}}\right) \text { moderately above } \mathrm{ID}_{50}\end{cases}
$$

Conclusion for Regime 2: The immunity caused by the preceding dose on the subsequent dose of moderately lower SELA value, is large and approaches $100 \%$.

We numerically demonstrate the asymptotic behaviors of regime 1 and regime 2. In Figure 6, we plot the immunity factor $\phi\left(S_{E_{1}}, S_{E_{2}}\right)$ vs $\left(S_{E_{2}}-S_{E_{1}}\right)$ and the two asymptotic solutions, respectively, for low $S_{E_{2}}-S_{E_{1}}$ and for high $S_{E_{2}}-S_{E_{1}}$. 


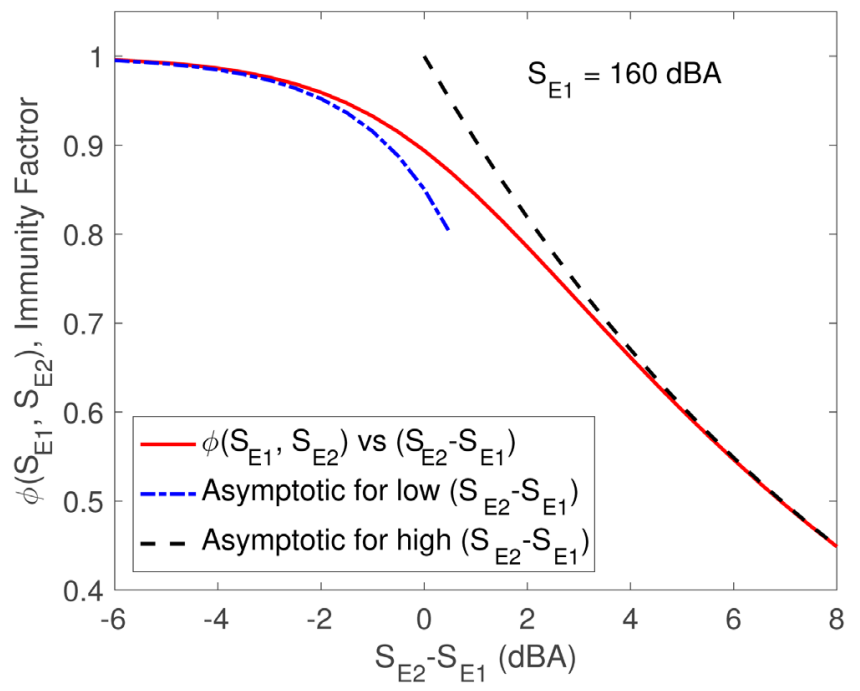

Figure 6. Immunity factor caused by preceding dose $S_{E_{1}}$ on subsequent dose $S_{E_{2}}$. Plot of $\phi\left(S_{E_{1}}, S_{E_{2}}\right)$ vs $\left(S_{E_{2}}-S_{E_{1}}\right)$ at $S_{E_{1}}=160 \mathrm{dBA}$, and asymptotic approximations respectively for low $\left(S_{E_{2}}-S_{E_{1}}\right)$ and for high $\left(S_{E_{2}}-S_{E_{1}}\right)$. The parameters used are $\lambda=3.44, \alpha=0.1, \quad \mathrm{ID}_{50}=170 \mathrm{dBA}$ from [6].

From Figure 6, it is clear that the two asymptotic approximations are already very accurate even at $S_{E_{2}}-S_{E_{1}}= \pm 4 \mathrm{dBA}$. Although only the plot at $S_{E_{1}}=160 \mathrm{dBA}$ is shown, we observe that the plot of $\phi\left(S_{E_{1}}, S_{E_{2}}\right)$ vs $\left(S_{E_{2}}-S_{E_{1}}\right)$ is almost invariant with respect to $S_{E_{1}}$; plots with different values of $S_{E_{1}}$ virtually coincide with each other (not shown in Figure 6). To see the invariance with respect to $S_{E_{1}}$, in Figure 7 we plot $\phi\left(S_{E_{1}}, S_{E_{2}}\right)$ vs $S_{E_{1}}$ respectively at several fixed values of $\left(S_{E_{2}}-S_{E_{1}}\right)$. Figure 7 confirms that $\phi\left(S_{E_{1}}, S_{E_{2}}\right)$ is virtually independent of $S_{E_{1}}$ provided that $S_{E_{2}}$ is varied along with $S_{E_{1}}$ to make the difference $\left(S_{E_{2}}-S_{E_{1}}\right)$ fixed. This observation corresponds to the result we obtain in Regime 3 below.

Regime 3: The two doses are close to each other.

$$
S_{E_{2}}-S_{E_{1}}=\log (O(1))
$$

which is equivalent to $\frac{W_{1}}{W_{2}}=O(1)$.

We start with the expression of the immunity factor $\phi$ given in (49) as a function of $\frac{W_{1}}{W_{2}}$ and $W_{2}$. In Regimes 1 and 2, we see that when the ratio $\frac{W_{1}}{W_{2}}$ is varied from small (Regime 1) to large (Regime 2), the immunity factor changes dramatically from $\phi \approx 0$ to $\phi \approx 100 \%$. When $\frac{W_{1}}{W_{2}}$ is fixed (Regime 3), however, the immunity factor $\phi$ varies much slower with respect to $W_{2}$. Now we prove that when the ratio $\frac{W_{1}}{W_{2}}$ is fixed, the immunity factor $\phi$ increases monotonically with respect to $W_{2}$. 


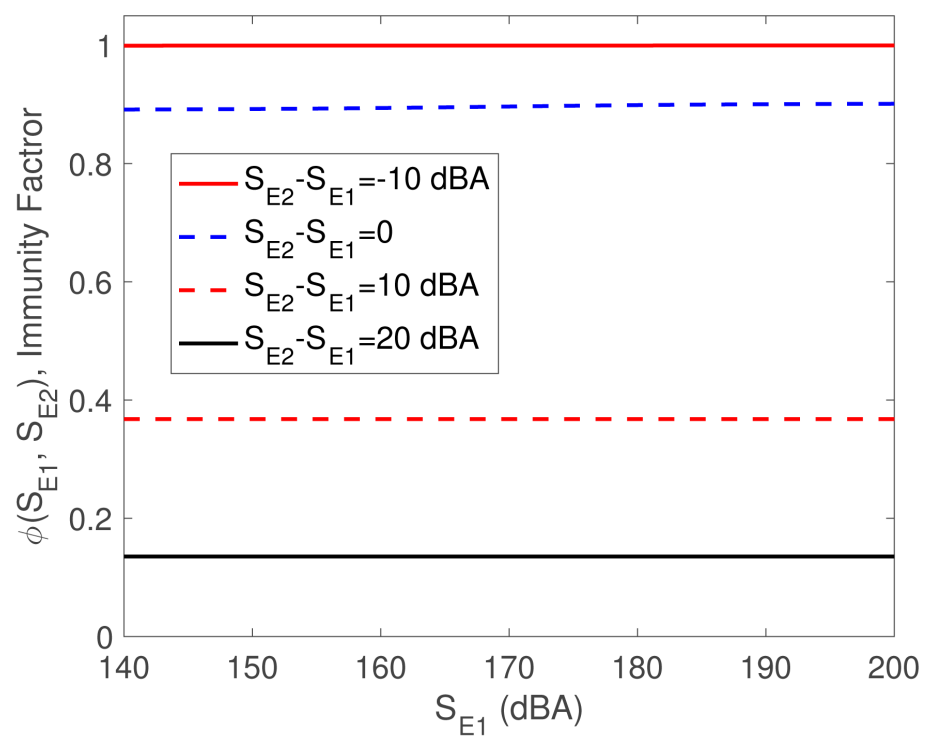

Figure 7. Immunity factor caused by preceding dose $S_{E_{1}}$ on subsequent dose $S_{E_{2}}$. Plot of $\phi\left(S_{E_{1}}, S_{E_{2}}\right)$ vs $S_{E_{1}}$ at several fixed values of $\left(S_{E_{2}}-S_{E_{1}}\right)$. While $\phi\left(S_{E_{1}}, S_{E_{2}}\right)$ varies widely from 0 to 1 with $\left(S_{E_{2}}-S_{E_{1}}\right)$, it is virtually independent of $S_{E_{1}}$. The parameters used are $\lambda=3.44, \alpha=0.1, \mathrm{ID}_{50}=170 \mathrm{dBA}$ from [6].

To facilitate the mathematical analysis, let us introduce variables

$$
r \equiv \frac{W_{1}}{W_{2}}, \quad u \equiv W_{2}^{\eta}
$$

The immunity factor as a function of $(r, u)$ has the expression

$$
\phi=\frac{r^{\eta}(1+u)+1-(1+r)^{\eta}}{1+u(1+r)^{\eta}}
$$

Taking the derivative of $\phi$ with respect to $u$, yields

$$
\frac{\mathrm{d} \phi}{\mathrm{d} u}=\frac{\left[(1+r)^{\eta}-r^{\eta}\right]\left[(1+r)^{\eta}-1\right]}{\left[1+u(1+r)^{\eta}\right]^{2}}>0
$$

This tells us immediately that the immunity factor $\phi$ is an increasing function of $W_{2}$. The range of $\phi$ with respect to $W_{2}$ is readily obtained from expression (54).

$$
\begin{gathered}
\left.\phi\right|_{W_{2}=0}=r^{\eta}+1-(1+r)^{\eta} \\
\left.\phi\right|_{W_{2}=\infty}=\frac{r^{\eta}}{(1+r)^{\eta}}
\end{gathered}
$$

In the case of $\eta=0.15$ and $r \equiv \frac{W_{1}}{W_{2}}=1$, the range $\left(\left.\phi\right|_{W_{2}=0},\left.\phi\right|_{W_{2}=\infty}\right)$ is fairly tight:

$$
\left.\phi\right|_{W_{2}=0}=2-2^{\eta}=0.8904
$$




$$
\left.\phi\right|_{W_{2}=\infty}=2^{-\eta}=0.9013
$$

Conclusion for Regime 3: The immunity caused by the preceding dose on the subsequent dose of similar magnitude (small difference measured in dBA), is significant and fairly large. The immunity factor remains almost unchanged as the two SELA values $\left(S_{E_{1}}\right.$ and $S_{E_{2}}$ ) are varied from being significantly below the median injury dose $\mathrm{ID}_{50}$ to being significantly above $\mathrm{ID}_{50}$ as long as the two doses are varied together with the difference $S_{E_{1}}-S_{E_{2}}$ fixed.

With the expression of $\phi$ in terms of $r \equiv \frac{W_{1}}{W_{2}}$ and $u \equiv W_{2}^{\eta}$ given in (54), we continue to explore the immunity factor $\phi$ as a function of $r$ while $u$ is fixed.

Regime 4: Instead of focusing on a small parameter region that gives a particular asymptotic value of the immunity factor, here we consider the trend behavior of the immunity factor when the first dose $\left(S_{E_{1}}\right)$ is varied from low to high while the second dose $\left(S_{E_{2}}\right)$ is fixed.

Differentiating (54) with respect to $r$, we get

$$
\frac{\mathrm{d} \phi}{\mathrm{d} r}=\eta(1+u) \frac{r^{\eta-1}-(1+r)^{\eta-1}+u r^{\eta-1}(1+r)^{\eta-1}}{\left[1+u(1+r)^{\eta}\right]^{2}}
$$

Since parameter $\eta$ is less than 1 , we have $\eta-1<0$, and it follows that $r^{\eta-1}-(1+r)^{\eta-1}>0$ holds for all positive $r$. Applying this result to the numerator of (57) gives us

$$
\frac{\mathrm{d} \phi}{\mathrm{d} r}>0, \quad \text { for } r>0 \text { and } u>0
$$

The range of $\phi$ with respect to $r=\frac{W_{1}}{W_{2}}$ is calculated from (54).

$$
\begin{aligned}
& \left.\phi\right|_{r=0}=0 \\
& \left.\phi\right|_{r=\infty}=1
\end{aligned}
$$

Therefore, we conclude that when $W_{2}$ is fixed, the immunity factor $\phi$ increases monotonically with respect to $W_{1}$. Below, we recast this statement in terms of the SELA values of the two events.

Conclusion for Regime 4: The immunity caused by the preceding dose on the subsequent dose increases monotonically with respect to the first dose while the second dose is fixed. It goes from near 0 when the first dose is significntly below the second dose, to near $100 \%$ when the first dose is raised to above the second dose.

We numerically visualize the trend behavior predicted in Regime 4. In Figure 8, we plot the immunity factor $\phi\left(S_{E_{1}}, S_{E_{2}}\right)$ as a function of the first dose $S_{E_{1}}$ while the second dose $S_{E_{2}}$ is fixed. Figure 8 shows that $\phi\left(S_{E_{1}}, S_{E_{2}}\right)$ is always an increasing function of $S_{E_{1}}$; for $S_{E_{1}}$ significantly below $S_{E_{2}}, \phi\left(S_{E_{1}}, S_{E_{2}}\right)$ is near zero; as $S_{E_{1}}$ approaches and goes above $S_{E_{2}}, \phi\left(S_{E_{1}}, S_{E_{2}}\right)$ climbs to its maximum value 1 . 


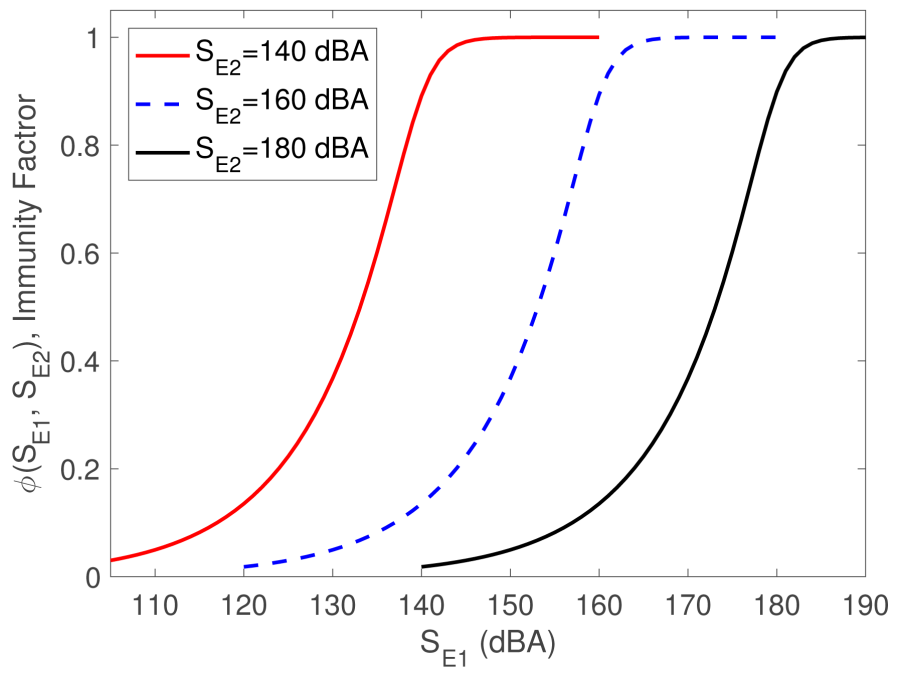

Figure 8. Immunity factor caused by preceding dose $S_{E_{1}}$ on subsequent dose $S_{E_{2}}$. Plot of $\phi\left(S_{E_{1}}, S_{E_{2}}\right)$ vs $S_{E_{1}}$ at several fixed values of $S_{E_{2}}$. The parameters used are $\lambda=3.44, \alpha=0.1, \quad \mathrm{ID}_{50}=170 \mathrm{dBA}$ from [6].

\section{Concluding Remarks}

We have theoretically examined the results in [6] to establish an extended empirical dose-response formulation that governs the probability of injury caused by multiple heterogeneous sound exposure events with non-uniform SELA values. The formulation consists of two steps: 1) the dose combination rule combines multiple heterogeneous doses into one effective dose; 2) the dose-response relation maps the effective dose to the injury probability. Our analysis revealed that in the empirical logistic dose-response relation with the empirical dose combination rule in [6], a pair of preceding and subsequent sound exposure events are not independent of each other; a preceding sound exposure always provides a net effect of immunity to the subsequent sound exposure. This result indicates that the interaction between preceding and subsequent events is dominated by the effect of immunity instead of by that of fatigue, suggesting that the acoustic reflex, for example, may play a significant role. Acoustic reflex is an involuntary muscle contraction in the middle ear triggered by high-intensity sound. During acoustic reflex, due to muscle contraction, the transmission of vibrational energy to the cochlea is reduced and the ear gets protected from the subsequent sound exposure. The effect of immunity was already implicitly contained (hidden) in the experimental data behind the empirical models in [6]. It was not hypothetically created or added in our study. Rather, our study simply discovered it through a detailed analysis on the empirical models, with the approach of viewing individual sound exposures as separate injury causing events. In the framework of empirical logistic dose-response relation in [6], we constructed the dose combination rule for the case where individual sound exposure events are independent of each other. We found that for a sequence of $N$ homogeneous impulse shots, the combined SELA 
value for the case of independent events is asymptotically a linear function of $N$ for large $N$. In comparison, the empirical dose combination rule is a linear function of $\log _{10}(N)$, much smaller than that for independent events.

We also studied the effect of immunity quantitatively via the immunity factor defined as the percentage of decrease in the injury probability attributed to the sound exposure in the preceding event. Our main results on immunity are: 1) the immunity factor is primarily a function of the difference in SELA value between the two sound exposure events; it is virtually independent of the magnitude of the two SELA values as long as the difference between the two is fixed; 2) the immunity factor increases monotonically with respect to the difference in SELA values; 3) when the first dose (SELA) is significantly below the second dose, the immunity factor is close to $0 ; 4)$ when the two doses are comparable, the immunity factor is fairly large, approaching 100\%; 5) when the first dose is moderately above the second dose, the immunity factor is close to $100 \%$.

Our extended empirical dose-response formulation provides the theoretical foundation for assessing the injury risk in realistic situations where the sound exposure consists of multiple heterogeneous noise impulses with non-uniform SELA values. Future sound exposure experiments are needed for testing, validating and refining the extended empirical dose-response formulation.

\section{Disclaimer}

The authors would like to thank Joint Non-Lethal Weapons Directorate of US Department of Defense for supporting this work. The views expressed in this document are those of the authors and do not reflect the official policy or position of the Department of Defense or the US Government.

\section{References}

[1] Hearing Loss Association of America. Types, Causes and Treatment. http://www.hearingloss.org/content/types-causes-and-treatment

[2] United States Government Accountability (2011) GAO-11-114: Hearing Loss Prevention: Improvements to DOD Hearing Conservation Programs Could Lead to Better Outcomes. Report to Congressional Committees, Washington DC.

[3] US Department of Veterans Affairs, Office of Research \& Development. VA Research on Hearing Loss. http://www.research.va.gov/topics/hearing.cfm

[4] Yankaskas, K. (2013) Prelude: Noise-Induced Tinnitus and Hearing Loss in the Military. Hearing Research, 295, 3-8. https://doi.org/10.1016/j.heares.2012.04.016

[5] Cho, S.I., Gao, S.S., Xia, A., Wang, R., Salles, F.T., et al. (2013) Mechanisms of Hearing Loss after Blast Injury to the Ear. PLoS ONE, 8, e67618. https://doi.org/10.1371/journal.pone.0067618

[6] Chan, P., Ho, K. and Ryan, A.F. (2016) Impulse Noise Injury Model. Military Medicine, 181, 59-69. https://doi.org/10.7205/MILMED-D-15-00139

[7] Hamernik, R.P., Patterson, J.H., Ahroon, W.A. and Stuhmiller, J.H. (1998) A Health Hazard Assessment for Blast Overpressure Exposures Subtitle-Use of Animal Test Data in the Development of a Human Auditory Hazard Criterion for Impulse 
Noise. Auditory Research Laboratory, State University of New York at Plattsburgh, Fort Detrick. http://www.dtic.mil/cgi-bin/GetTRDoc?AD=ADA393522

[8] Weightman, F.L., Flamme, G., Campenella, A.J. and Luz, G.A. (2010) American Institute of Biological Sciences Peer Review of Injury Prevention and Reduction Research Task Area-Impulse Noise Injury Models.

http://www.arl.army.mil/www/pages/343/AHAAH_AIBS_revew_Public_Release_11 Aug14.pdf

[9] Chan, P.C., Ho, K.H., Kan, K.K., Stuhmiller, J.H. and Mayorga, M.A. (2001) Evaluation of Impulse Noise Criteria using Human Volunteer Data. The Journal of the Acoustical Society of America, 10, 1967-1975. https://doi.org/10.1121/1.1391243

[10] Johnson, D.L. (1998) Blast Overpressure Studies. Technical Report USAARL-CR98-03. U.S. Army Aeromedical Research Laboratory, Fort Rucker.

http://www.dtic.mil/cgi-bin/GetTRDoc?AD=ADA347634

\section{Appendix}

Here we give a proof of Theorem 1 appeared in Section 3.

For $\eta \equiv \frac{\alpha \lambda}{\ln (10)}<1$, we have $(\eta-1)<0$. Consider function

$$
g(w)=W_{E_{1}}^{\eta}+w^{\eta}-\left(W_{E_{1}}+w\right)^{\eta}
$$

It satisfies

$$
\begin{gathered}
g(0)=0 \\
g^{\prime}(w)=\eta\left[w^{\eta-1}-\left(W_{E_{1}}+w\right)^{\eta-1}\right]>0 \text { for all } w>0
\end{gathered}
$$

It follows that $g(w)>0$ for all $w>0$. Or, equivalently,

$$
W_{E_{1}}^{\eta}+W_{E_{2}}^{\eta}-\left(W_{E_{1}}+W_{E_{2}}\right)^{\eta}>0 \text { for all } W_{E_{2}}>0\left(\text { and } W_{E_{1}}>0\right)
$$

From this, we see that

$$
W_{E_{1}}^{\eta}+W_{E_{2}}^{\eta}-\left(W_{E_{1}}+W_{E_{2}}\right)^{\eta}+W_{E_{1}}^{\eta} W_{E_{2}}^{\eta}>0 \text { for all } W_{E_{1}}>0 \text { and } W_{E_{2}}>0
$$

which completes the derivation. 